\title{
Common Fixed Point Theorems on Generalized Ratio Contraction Mapping in Extended Rectangular b-Metric Spaces
}

\author{
Budi Nurwahyu \\ Department of Mathematics, Hasanuddin University, Tamalanrea KM 10, Makassar, Indonesia \\ Correspondence should be addressed to Budi Nurwahyu; budinurwahyu@unhas.ac.id
}

Received 7 October 2019; Accepted 12 November 2019; Published 1 December 2019

Academic Editor: Ram U. Verma

Copyright (c) 2019 Budi Nurwahyu. This is an open access article distributed under the Creative Commons Attribution License, which permits unrestricted use, distribution, and reproduction in any medium, provided the original work is properly cited.

In this paper, we propose and prove the common fixed point theorems on generalized contraction mappings in extended rectangular $b$-metric spaces by utilizing the weakly compatible function property.

\section{Introduction}

The notion of $b$-metric space was introduced by Czerwik in 1993 [1], as a generalization of a metric space, by modifying the third condition in the metric space. Mohanta [2] and Husain et al. [3] utilized that space to prove existence and uniqueness of common fixed points. Recently, Kamran et al. [4] in 2017 generalized $b$-metric to become extended $b$-metric for utilizing in fixed point results and Alqahtani et al. [5] in 2018 utilized extended $b$ metric to prove the common fixed point. In 2015, George et al. [6] introduced the notion of rectangular $b$-metric space as a generalization of rectangular metric space. Kadelburg and Radenović [7] and Mitrovic [8] established and proved the common fixed point in such space. Very recently, Asim et al. [9] in 2019 generalized the rectangular $b$-metric and termed it the extended rectangular $b$-metric. By utilizing this space, Mustafa et al. [10] proved some fixed point results for contracting mappings.

Inspired of some fixed point results in extended rectangular $b$-metric, in the main results, we establish and prove existence and uniqueness of the common fixed point in the extended rectangular $b$-metric and furnish examples to clarify the results.

\section{Preliminaries}

In the following section, we need some definitions to govern our theorems.
Definition 1 (see [1]). Let $X$ be a nonempty set. A mapping $d_{b}: X \times X \longrightarrow[0, \infty)$ is called a $b$-metric, if there exists $b \geq 1$ such that $d_{b}$ satisfies the following conditions:

(1) $d_{b}(x, y)=0$, if and only if $x=y$

(2) $d_{b}(x, y)=d_{b}(y, x)$

(3) $d_{b}(x, y) \leq b\left[d_{b}(x, s)+d_{b}(s, y)\right]$

for all $x, y, s \in X$.

The pair $\left(X, d_{b}\right)$ is called a $b$-metric space.

Definition 2 (see [4]). Let $X$ be a nonempty set. A mapping $d_{b}: X \times X \longrightarrow[0, \infty)$ is called an extended $b$-metric, if there exists a function $b: X \times X \longrightarrow[1, \infty)$ such that $d_{b}$ satisfies the following conditions:

(1) $d_{b}(x, y)=0$, if and only if $x=y$

(2) $d_{b}(x, y)=d_{b}(y, x)$

(3) $d_{b}(x, y) \leq b(x, y)\left[d_{b}(x, s)+d_{b}(s, y)\right]$

for all $x, y, s \in X$.

The pair $\left(X, d_{b}\right)$ is called an extended b-metric space.

Definition 3 (see [6]). Let $X$ be a nonempty set. A mapping $d_{b}: X \times X \longrightarrow[0, \infty)$ is called rectangular $b$-metric, if there exists $b \geq 1$ such that $d_{b}$ satisfies the following conditions:

(1) $d_{b}(x, y)=0$, if and only if $x=y$

(2) $d_{b}(x, y)=d_{b}(y, x)$ 
(3) $d_{b}(x, y) \leq b\left[d_{b}(x, s)+d_{b}(s, t)+d_{b}(t, y)\right]$

for all $x, y \in X$ and $s, t \in X \backslash\{x, y\}$.

The pair $\left(X, d_{b}\right)$ is called a rectangular b-metric space.

Definition 4 (see [9]). Let $X$ be a nonempty set. A mapping $d_{b}: X \times X \longrightarrow[0, \infty)$ is called an extended rectangular $b$-metric, if there exists a function $b: X \times X \longrightarrow[1, \infty)$ such that $d_{b}$ satisfies the following conditions:

$$
\begin{aligned}
& \text { (A1) } d_{b}(x, y)=0, \quad \text { if and only if } x=y, \\
& \text { (A2) } d_{b}(x, y)=d_{b}(y, x), \\
& \text { (A3) } d_{b}(x, y) \leq b(x, y)\left[d_{b}(x, s)+d_{b}(s, t)+d_{b}(t, y)\right]
\end{aligned}
$$

for all $x, y \in X$ and $s, t \in X \backslash\{x, y\}$.

The pair $\left(X, d_{b}\right)$ is called an extended rectangular $b$-metric space.

Example 1. Let $X=[0, \infty)$ and $d_{b}(x, y)=(x-y)^{p}$, and $p>1$ with $b(x, y)=x+y+3^{p-1}$.

It is obvious for condition A1 and A2. For condition A3, we consider from the Jensen inequality.

Thus, we have $d_{b}(x, y)=(x-y)^{2} \leq 3^{p-1}\left[(x-s)^{2}+\right.$ $\left.(s-t)^{2}+(t-y)^{2}\right] \leq\left(x+y+3^{p-1}\right)\left[(x-s)^{2}+(s-t)^{2}+(t-\right.$ $\left.y)^{2}\right]=b(x, y)\left[d_{b}(x, s)+d_{b}(s, t)+d_{b}(t, y)\right]$ for all $x, y$, $s, t \in X$

So, this shows that $d_{b}(x, y)=(x-y)^{p}$ is an extended rectangular $b$-metric with $b(x, y)=x+y+3^{p-1}$. However, in general $d_{b}(x, y)$ is not continuous.

Example 2 (see [7]). Let $X=\{0,2\} \cup\{1 / n \mid n \in N\}$, and choose a function $b(x, y)=x+y+2$, for $x, y \in X$. Define $d_{b}: X \times X \longrightarrow[0, \infty)$ a function as follows:

$$
d_{b}(x, y)= \begin{cases}0, & x=y, \\ 1, & x \neq y, x, y \in\{0,2\} \text { or } x, y \in\left\{\frac{1}{n} \mid n \in N\right\}, \\ y, & x \in\{0,2\}, y \in\left\{\frac{1}{n} \mid n \in N\right\}, \\ x, & x \in\left\{\frac{1}{n} \mid n \in N\right\}, y \in\{0,2\} .\end{cases}
$$

It is easy to show that $d_{b}$ is the complete extended rectangular $b$-metric on $X$ with $b(x, y)=x+y+2$.

If we choose a sequence $\{1 / n\}_{n \in N}$, it is easy to show that sequence $\{1 / n\}_{n \in N}$ is convergent to 0 and 2 , so it is not a
Cauchy sequence. Moreover, $d_{b}$ is not continuous, since $\lim _{n \longrightarrow \infty}(1 / n)=0$ and $1=\lim _{n \rightarrow \infty} d_{b}(1 / n, 1 / 3) \neq d_{b}(0$, $(1 / 3))=1 / 3$. Thus, $\left(X, d_{b}\right)$ is not metrizable because there exists a sequence in $\left(X, d_{b}\right)$ that is not convergent to unique point in $X$.

Our proofs in the main results need uniqueness of the limit point of sequence in $\left(X, d_{b}\right)$ as given in the following lemma.

Lemma 1 (see $[9,11])$. Let $\left(X, d_{b}\right)$ be a complete extended rectangular b-metric space and $\left\{y_{n}\right\}$ is a Cauchy sequence. If $y_{n} \neq y_{m}$ for all $n \neq m$, then limit point of $\left\{y_{n}\right\}$ is unique.

Definition 5 (see [12]). Let $X$ be a nonempty set and $T_{1}, T_{2}$ : $X \longrightarrow X$ be self-mapping. If $T_{1} x=T_{2} x=y$ for some $x \in X$, then $\mathrm{y}$ is called a point of coincidence of $T_{1}$ and $T_{2}$ and $\mathrm{x}$ is called a coincidence point of $T_{1}$ and $T_{2}$.

Definition 6 (see [13]). Let $X$ be a nonempty set and $T_{1}, T_{2}$ : $X \longrightarrow X$ be self-mappings. $\left\{T_{1}, T_{2}\right\}$ is called weakly compatible. For every $x \in X$, if $T_{1} x=T_{2} x$, then $T_{2} T_{1} x=T_{1} T_{2} x$.

Definition 7 (see [9]). Let $\left(X, d_{b}\right)$ be an extended rectangular $b$-metric space, then sequence

(a) $\left\{x_{n}\right\}$ is called convergent if and only if there exists $x \in X$ such that $d_{b}\left(x_{n}, x\right) \longrightarrow 0$, as $n \longrightarrow \infty$.

(b) $\left\{x_{n}\right\}$ is called Cauchy if and only if $d_{b}\left(x_{n}, x_{m}\right) \longrightarrow 0$, as $n, m \longrightarrow \infty$.

In addition, $X$ is called complete if every Cauchy sequence in $X$ is convergent in $X$.

\section{Main Results}

In this section, we establish and prove the common fixed point theorems in complete extended rectangular $b$ metric space and furnish some examples to clarify the theorem.

Lemma 2. Let $\left(X, d_{b}\right)$ be an extended rectangular b-metric space and $\left\{y_{n}\right\}$ be a sequence in $X$.

If $d_{b}\left(y_{n}, y_{n+1}\right) \leq K d_{b}\left(y_{n-1}, y_{n}\right), \quad 0<K<1, \quad$ and $\lim _{n, m \rightarrow \infty} b\left(y_{n}, y_{m}\right)<(1 / K)$, then $\left\{y_{n}\right\}$ is a Cauchy.

Proof. Let $m \in N, m>n$.

Case 1. For $m-n$ is odd, let $m-n=2 k+1$. 
Consider that from rectangular condition in (1) we have

$$
\begin{aligned}
& d_{b}\left(y_{n}, y_{m}\right) \leq b\left(y_{n}, y_{m}\right)\left[d_{b}\left(y_{n}, y_{n+1}\right)+d_{b}\left(y_{n+1}, y_{n+2}\right)+d_{b}\left(y_{n+2}, y_{m}\right)\right] \\
& \leq b\left(y_{n}, y_{m}\right)\left[d_{b}\left(y_{n}, y_{n+1}\right)+d_{b}\left(y_{n+1}, y_{n+2}\right)\right]+b\left(y_{n}, y_{m}\right) d_{b}\left(y_{n+2}, y_{m}\right) \\
& \leq b\left(y_{n}, y_{m}\right)\left[K^{n} d_{b}\left(y_{0}, y_{1}\right)+K^{n+1} d_{b}\left(y_{0}, y_{1}\right)\right]+b\left(y_{n}, y_{m}\right) d_{b}\left(y_{n+2}, y_{m}\right) \\
& \leq\left(K^{n}+K^{n+1}\right) d_{b}\left(y_{0}, y_{1}\right) b\left(y_{n}, y_{m}\right)+\left(K^{n+2}+K^{n+3}\right) d_{b}\left(y_{0}, y_{1}\right) b\left(y_{n}, y_{m}\right) b\left(y_{n+2}, y_{m}\right) \\
& +b\left(y_{n}, y_{m}\right) b\left(y_{n+2}, y_{m}\right) d_{b}\left(y_{n+4}, y_{m}\right) \\
& \leq\left(K^{n}+K^{n+1}\right) d_{b}\left(y_{0}, y_{1}\right) b\left(y_{n}, y_{m}\right) \\
& +\left(K^{n+2}+K^{n+3}\right) d_{b}\left(y_{0}, y_{1}\right) b\left(y_{n}, y_{m}\right) b\left(y_{n+2}, y_{m}\right)+\cdots \\
& +\left(K^{n+2 k-2}+K^{n+2 k-1}\right) d_{b}\left(y_{0}, y_{1}\right) b\left(y_{n}, y_{m}\right) b\left(y_{n+2}, y_{m}\right) \cdots b\left(y_{n+2 k-2}, y_{m}\right)+b\left(y_{n}, y_{m}\right) b\left(y_{n+2}, y_{m}\right) \cdots b \\
& \cdot\left(y_{n+2 k-2}, y_{m}\right) d_{b}\left(y_{n+2 k}, y_{m}\right) \\
& +\left(K^{n+2 k-2}+K^{n+2 k-1}\right) d_{b}\left(y_{0}, y_{1}\right) b\left(y_{n}, y_{m}\right) b\left(y_{n+2}, y_{m}\right) \cdots b\left(y_{n+2 k-2}, y_{m}\right)+b\left(y_{n}, y_{m}\right) b\left(y_{n+2}, y_{m}\right) \cdots b \\
& \cdot\left(y_{n+2 k-2}, y_{m}\right) d_{b}\left(y_{n+2 k}, y_{m}\right) \\
& \leq\left(K^{n}+K^{n+1}\right) d_{b}\left(y_{0}, y_{1}\right) b\left(y_{n}, y_{m}\right) \\
& +\left(K^{n+2}+K^{n+3}\right) d_{b}\left(y_{0}, y_{1}\right) b\left(y_{n}, y_{m}\right) b\left(y_{n+2}, y_{m}\right)+\cdots \\
& +\left(K^{n+2 k-2}+K^{n+2 k-1}\right) d_{b}\left(y_{0}, y_{1}\right) b\left(y_{n}, y_{m}\right) b\left(y_{n+2}, y_{m}\right) \cdots b\left(y_{n+2 k-2}, y_{m}\right)+\cdots+b\left(y_{n}, y_{m}\right) b\left(y_{n+2}, y_{m}\right) \cdots b \\
& \cdot\left(y_{n+2 k-2}, y_{m}\right) K^{n+2 k} d_{b}\left(y_{0}, y_{1}\right) \\
& =(1+K) d_{b}\left(y_{0}, y_{1}\right) K^{n} \sum_{j=0}^{k-1} K^{2 j} \prod_{i=0}^{j} b\left(y_{n+2 i}, y_{m}\right)+b\left(y_{n}, y_{m}\right) b\left(y_{n+2}, y_{m}\right) \cdots b\left(y_{n+2 k-2}, y_{m}\right) K^{n+2 k} d_{b}\left(y_{0}, y_{1}\right) \text {. }
\end{aligned}
$$

Since $b\left(y_{i}, y_{j}\right) \geq 1$, using (3) we have that

$$
\begin{aligned}
d_{b}\left(y_{n}, y_{m}\right) & \leq(1+K) d_{b}\left(y_{0}, y_{1}\right)\left\{K^{n}\left(\sum_{j=0}^{k-1} K^{2 j} \prod_{i=0}^{j} b\left(y_{n+2 i}, y_{m}\right)+b\left(y_{n}, y_{m}\right) b\left(y_{n+2}, y_{m}\right) \cdots b\left(y_{n+2 k-2}, y_{m}\right) K^{2 k}\right)\right\} \\
& \leq(1+K) d_{b}\left(y_{0}, y_{1}\right)\left\{K^{n}\left(\sum_{j=n}^{m-1} K^{j-n} \prod_{i=1}^{j} b\left(y_{i}, y_{m}\right)\right)\right\}=(1+K) d_{b}\left(y_{0}, y_{1}\right)\left(\sum_{j=n}^{m-1} K^{j} \prod_{i=1}^{j} b\left(y_{i}, y_{m}\right)\right) \\
& =(1+K) d_{b}\left(y_{0}, y_{1}\right)\left(S_{m-1}-S_{n-1}\right),
\end{aligned}
$$

where $S_{n}=\sum_{j=0}^{n} K^{j} \prod_{i=1}^{j} b\left(y_{i}, y_{m}\right)$.

Let $a_{j}=K^{j} \prod_{i=1}^{j} b\left(y_{i}, y_{m}\right)$, so by applying ratio criteria and as known that $\lim _{n, m \rightarrow \infty} b\left(y_{n}, y_{m}\right)<(1 / K)$, then we have $\lim _{j \longrightarrow \infty}\left(a_{j+1} / a_{j}\right)=\lim _{j \longrightarrow \infty} K b\left(y_{j}, y_{m}\right)<1$ for each $m \in N$.
This implies that $S_{n}=\sum_{j=1}^{n} K^{j} \prod_{i=1}^{j} b\left(y_{i}, y_{m}\right)$ is convergent for each $m \in N$.

Hence, for $n, m \longrightarrow \infty$ in (4) we get $d_{b}\left(y_{n}, y_{m}\right) \longrightarrow 0$; thus, $\left\{y_{n}\right\}$ is the Cauchy sequence in $X$. 
Case 2. For $m-n$ is even, let $m-n=2 k$.

$$
\begin{aligned}
d_{b}\left(y_{n}, y_{m}\right) \leq & b\left(y_{n}, y_{m}\right)\left[d_{b}\left(y_{n}, y_{n+1}\right)+d_{b}\left(y_{n+1}, y_{n+2}\right)+d_{b}\left(y_{n+2}, y_{m}\right)\right] \\
\leq & b\left(y_{n}, y_{m}\right)\left[d_{b}\left(y_{n}, y_{n+1}\right)+d_{b}\left(y_{n+1}, y_{n+2}\right)\right]+b\left(y_{n}, y_{m}\right) d_{b}\left(y_{n+2}, y_{m}\right) \\
\leq & b\left(y_{n}, y_{m}\right)\left[K^{n} d_{b}\left(y_{0}, y_{1}\right)+K^{n+1} d_{b}\left(y_{0}, y_{1}\right)\right]+b\left(y_{n}, y_{m}\right) d_{b}\left(y_{n+2}, y_{m}\right) \\
\leq & \left(K^{n}+K^{n+1}\right) d_{b}\left(y_{0}, y_{1}\right) b\left(y_{n}, y_{m}\right)+b\left(y_{n}, y_{m}\right) d_{b}\left(y_{n+2}, y_{m}\right) \\
\leq & \left(K^{n}+K^{n+1}\right) d_{b}\left(y_{0}, y_{1}\right) b\left(y_{n}, y_{m}\right) \\
& +b\left(y_{n}, y_{m}\right) b\left(y_{n+2}, y_{m}\right)\left[d_{b}\left(y_{n+2}, y_{n+3}\right)+d_{b}\left(y_{n+3}, y_{n+4}\right)\right] \\
& +b\left(y_{n}, y_{n+2 k}\right) b\left(y_{n+2}, y_{m}\right) d_{b}\left(y_{n+4}, y_{m}\right) \\
\leq & \left(K^{n}+K^{n+1}\right) d_{b}\left(y_{0}, y_{1}\right) b\left(y_{n}, y_{m}\right) \\
& +b\left(y_{n}, y_{m}\right) b\left(y_{n+2}, y_{m}\right)\left[K^{n+2} d_{b}\left(y_{0}, y_{1}\right)+K^{n+3} d_{b}\left(y_{0}, y_{1}\right)\right] \\
& +b\left(y_{n}, y_{m}\right) b\left(y_{n+2}, y_{m}\right) d_{b}\left(y_{n+4}, y_{m}\right) \\
\leq & \left(K^{n}+K^{n+1}\right) d_{b} d_{b}\left(y_{0}, y_{1}\right) b\left(y_{n}, y_{m}\right) \\
& +\left(K^{n+2}+K^{n+3}\right) d_{b}\left(y_{0}, y_{1}\right) b\left(y_{n}, y_{m}\right) b\left(y_{n+2}, y_{m}\right)+\cdots \\
& +\left(K^{n+2 k-2}+K^{n+2 k-1}\right) d_{b}\left(y_{0}, y_{1}\right) b\left(y_{n}, y_{m}\right) b\left(y_{n+2}, y_{m}\right) \ldots b\left(y_{m-2}, y_{m}\right) \\
& +K^{n+2 k-2} d_{b}\left(y_{0}, y_{2}\right) b\left(y_{n}, y_{m}\right) b\left(y_{n+2}, y_{m}\right) \cdots b\left(y_{m-2}, y_{m}\right) \\
= & (1+K) d_{b}\left(y_{0}, y_{1}\right) K^{n} \sum_{j=1}^{j} K^{2 j} \prod_{i=0}^{j} b\left(y_{n+2 i}, y_{m}\right)+K^{m-2} d_{b}\left(y_{0}, y_{2}\right) \prod_{i=0}^{k-1} b\left(y_{n+2 i}, y_{m}\right) .
\end{aligned}
$$
have

Let $L=\max \left\{d_{b}\left(y_{0}, y_{1}\right), d_{b}\left(y_{0}, y_{2}\right)\right\}$, so by using (5) we

$$
\begin{aligned}
d_{b}\left(y_{n}, y_{m}\right) & \leq(1+K) L\left(K^{n} \sum_{j=1}^{k-1} K^{2 j} \prod_{i=0}^{j} b\left(y_{n+2 i}, y_{m}\right)+K^{m-2} \prod_{i=0}^{k-1} b\left(y_{n+2 i}, y_{m}\right)\right) \\
& =(1+K) L\left(K^{n} \sum_{j=1}^{k-1} K^{2 j} \prod_{i=0}^{j} b\left(y_{n+2 i}, y_{m}\right)+K^{n+2 k-2} \prod_{i=0}^{k-1} b\left(y_{n+2 i}, y_{m}\right)\right) \\
& =(1+K) L\left(K^{n}\left[\sum_{j=1}^{k-1} K^{2 j} \prod_{i=0}^{j} b\left(y_{n+2 i}, y_{m}\right)+K^{2 k-2} \prod_{i=0}^{k-1} b\left(y_{n+2 i}, y_{m}\right)\right]\right) \\
& \leq(1+K) L\left(K^{n}\left(\sum_{j=n}^{m-1} K^{j-n} \prod_{i=1}^{j} b\left(y_{i}, y_{m}\right)\right)\right)=(1+K) L\left(\sum_{j=n}^{m-1} K^{j} \prod_{i=1}^{j} b\left(y_{i}, y_{m}\right)\right) \\
& =(1+K) L\left(\sum_{j=0}^{m-1} K^{j} \prod_{i=1}^{j} b\left(y_{i}, y_{m}\right)-\sum_{j=0}^{n-1} K^{j} \prod_{i=1}^{j} b\left(y_{i}, y_{m}\right)\right)=(1+K) L\left(S_{m-1}-S_{n-1}\right) .
\end{aligned}
$$

This implies $d_{b}\left(y_{n}, y_{m}\right) \longrightarrow 0$ as $m, n \longrightarrow \infty$, thus $\left\{y_{n}\right\}$ is Cauchy in $X$.
Theorem 1. Let $\left(X, d_{b}\right)$ be a complete extended rectangular $b$-metric space and let $f, g: X \longrightarrow X$ be self-mappings such that $f(X) \subseteq g(X)$ and $g(X)$ closed. If

$$
b(x, y) d_{b}(f x, f y) \leq \frac{p d_{b}(g x, g y)+q d_{b}(g x, f x)+r(g y, f y)+s \beta d_{b}(f x, g y) d_{b}(f y, g x)}{\lambda b(f x, g x)+\beta\left(d_{b}(f x, g y)+d_{b}(f y, g x)\right)}
$$


holds for all $x, y \in X, \quad \beta>0, \quad 0<s<1, \quad 0<p+q+$ $r<\lambda, \quad(p / 1-s)<\lambda, \quad$ and $\quad \lim _{n, m \rightarrow \infty} b\left(y_{n}, y_{m}\right)<(\lambda-$ $r / p+q)$, then $f$ and $g$ have a unique coincidence in $X$.

Moreover, if $f$ and $g$ are weakly compatible, then $f$ and $g$ have a unique common fixed point.
Proof. Let $x_{0} \in X$ as an initial point, define sequence $\left\{x_{n}\right\}$ and $\left\{y_{n}\right\}$ in $X$ such that $y_{n}=f\left(x_{n}\right)=g\left(x_{n+1}\right)$, $n=0,1,2, \ldots$. Suppose that $y_{n} \neq y_{n+1}, n=0,1,2, \ldots$, because if $y_{n}=y_{n+1}$ for some $n$, then $g\left(x_{n+1}\right)=y_{n}=y_{n+1}=$ $f\left(x_{n+1}\right)$, so $f$ and $g$ have a coincidence point.

Considering (7), we have

$$
\begin{aligned}
b\left(x_{n}, x_{n+1}\right) d_{b}\left(y_{n}, y_{n+1}\right) & =b\left(x_{n}, x_{n+1}\right) d_{b}\left(f x_{n}, f x_{n+1}\right) \\
& \leq \frac{p d_{b}\left(g x_{n}, g x_{n+1}\right)+q d_{b}\left(g x_{n}, f x_{n}\right)+r d_{b}\left(g x_{n+1}, f x_{n+1}\right)+s \beta d_{b}\left(f x_{n}, g x_{n+1}\right) d_{b}\left(f x_{n+1}, g x_{n}\right)}{\lambda b\left(f x_{n}, g x_{n}\right)+\beta\left(d_{b}\left(f x_{n}, g x_{n+1}\right)+d_{b}\left(f x_{n+1}, g x_{n}\right)\right)} \\
& =\frac{p d_{b}\left(y_{n-1}, y_{n}\right)+q d_{b}\left(y_{n-1}, y_{n}\right)+r d_{b}\left(y_{n}, y_{n+1}\right)+s \beta d_{b}\left(y_{n}, y_{n}\right) d_{b}\left(y_{n+1}, y_{n}\right)}{\lambda b\left(y_{n}, y_{n-1}\right)+\beta\left(d_{b}\left(y_{n}, y_{n}\right)+d_{b}\left(y_{n+1}, y_{n}\right)\right)} \\
& =\frac{(p+q) d_{b}\left(y_{n-1}, y_{n}\right)+r d_{b}\left(y_{n}, y_{n+1}\right)}{\lambda b\left(y_{n}, y_{n-1}\right)+\beta d_{b}\left(y_{n+1}, y_{n}\right)} \\
& \leq \frac{(p+q) d_{b}\left(y_{n-1}, y_{n}\right)+r d_{b}\left(y_{n}, y_{n+1}\right)}{\lambda b\left(y_{n}, y_{n-1}\right)} .
\end{aligned}
$$

Since $\left(x_{n}, x_{n+1}\right) \geq 1$, then we have

$$
\begin{aligned}
d_{b}\left(y_{n}, y_{n+1}\right) & \leq \frac{(p+q) d_{b}\left(y_{n-1}, y_{n}\right)+r d_{b}\left(y_{n}, y_{n+1}\right)}{\lambda b\left(x_{n}, x_{n+1}\right) b\left(y_{n}, y_{n-1}\right)} \\
& \leq \frac{(p+q) d_{b}\left(y_{n-1}, y_{n}\right)+r d_{b}\left(y_{n}, y_{n+1}\right)}{\lambda},
\end{aligned}
$$

Thus, we get

$$
d_{b}\left(y_{n}, y_{n+1}\right) \leq \delta d_{b}\left(y_{n-1}, y_{n}\right)
$$

where $\delta=p+q / \lambda-r$. Since $p+q+r<\lambda$, we have $0<\delta<1$, and as known that $\lim _{n, m \rightarrow \infty} b\left(y_{n}, y_{m}\right)<1 / \delta$, then by using Lemma 2, we get $\left\{y_{n}\right\}$ is a Cauchy sequence in $g X$.

Since $X$ is complete, then there exists $u^{*} \in X$ such that

$$
d_{b}\left(y_{n}, u^{*}\right) \longrightarrow 0,
$$

and since $y_{n}=g\left(x_{n+1}\right) \subseteq X$ and $g X$ is a closed set, then $u^{*} \in g X$ and $u^{*}=g\left(x^{*}\right)$ for some $x^{*} \in X$.

Furthermore, we will show that $f$ and $g$ have a point coincidence as follows.

From (1) and (7) we have

$$
\begin{aligned}
d_{b}\left(f x^{*}, g x^{*}\right) \leq & b\left(f x^{*}, g x^{*}\right)\left[d_{b}\left(f x^{*}, y_{n}\right)+d_{b}\left(y_{n}, y_{n+1}\right)+d_{b}\left(y_{n+1}, g x^{*}\right)\right] \\
= & b\left(f x^{*}, g x^{*}\right)\left[d_{b}\left(f x^{*}, f x_{n}\right)+d_{b}\left(y_{n}, y_{n+1}\right)+d_{b}\left(y_{n+1}, u^{*}\right)\right] \\
\leq & b\left(f x^{*}, g x^{*}\right)\left[\frac{1}{b\left(x^{*}, x_{n}\right)}\left(\frac{p d_{b}\left(g x^{*}, g x_{n}\right)+q d_{b}\left(g x^{*}, f x^{*}\right)+r d_{b}\left(g x_{n}, f x_{n}\right)+s \beta d_{b}\left(f x^{*}, g x_{n}\right) d_{b}\left(f x_{n}, g x^{*}\right)}{\lambda b\left(f x^{*}, g x^{*}\right)+\beta\left(d_{b}\left(f x^{*}, g x_{n}\right)+d_{b}\left(f x_{n}, g x^{*}\right)\right)}\right)\right. \\
& \left.+d_{b}\left(y_{n}, y_{n+1}\right)+d_{b}\left(y_{n+1}, u^{*}\right)\right] \\
\leq & b\left(f x^{*}, g x^{*}\right)\left[\left(\frac{p d_{b}\left(u^{*}, y_{n-1}\right)+q d_{b}\left(u^{*}, f x^{*}\right)+r d_{b}\left(y_{n-1}, y_{n}\right)+s \beta d_{b}\left(f x^{*}, y_{n-1}\right) d_{b}\left(y_{n}, u^{*}\right)}{\lambda b\left(f x^{*}, u^{*}\right)+\beta\left(d_{b}\left(f x^{*}, y_{n-1}\right)+d_{b}\left(y_{n}, u^{*}\right)\right)}\right)\right. \\
& \left.+d_{b}\left(y_{n}, y_{n+1}\right)+d_{b}\left(y_{n+1}, u^{*}\right)\right] \\
\leq & b\left(f x^{*}, g x^{*}\right)\left[\left(\frac{p d_{b}\left(u^{*}, y_{n-1}\right)+q d_{b}\left(u^{*}, f x^{*}\right)+r d_{b}\left(y_{n-1}, y_{n}\right)}{\lambda b\left(f x^{*}, u^{*}\right)+\beta\left(d_{b}\left(f x^{*}, y_{n-1}\right)+d_{b}\left(y_{n}, u^{*}\right)\right)}+s d_{b}\left(y_{n}, u^{*}\right)\right)+d_{b}\left(y_{n}, y_{n+1}\right)+d_{b}\left(y_{n+1}, u^{*}\right)\right] \\
\leq & b\left(f x^{*}, g x^{*}\right)\left[\left(\frac{p d_{b}\left(u^{*}, y_{n-1}\right)+q d_{b}\left(u^{*}, f x^{*}\right)+r d_{b}\left(y_{n-1}, y_{n}\right)}{\lambda b\left(f x^{*}, u^{*}\right)}+s d_{b}\left(y_{n}, u^{*}\right)\right)+d_{b}\left(y_{n}, y_{n+1}\right)+d_{b}\left(y_{n+1}, u^{*}\right)\right] .
\end{aligned}
$$


It follows from (11) to (12) that if $n \longrightarrow \infty$, then we get $d_{b}\left(f x^{*}, g x^{*}\right) \leq \frac{b\left(f x^{*}, g x^{*}\right) d_{b}\left(u^{*}, f x^{*}\right)}{\lambda b\left(f x^{*}, g x^{*}\right)} \leq \frac{d_{b}\left(g x^{*}, f x^{*}\right)}{\lambda}$.
This implies that $d_{b}\left(f x^{*}, g x^{*}\right)=0$, so then we get $f x^{*}=g x^{*}=u^{*}$. Hence, $u^{*}$ is a coincidence point of $f$ and $g$.

To show uniqueness of the coincidence point of $f$ and $g$, we suppose there exists $w^{*} \in X$ such that $f y^{*}=g y^{*}=w^{*}$.

From (1) and (7), we have

$$
\begin{aligned}
d_{b}\left(u^{*}, w^{*}\right) & \left.=d_{b}\left(\left(f x^{*}, f y^{*}\right)\right\}\right) \\
& \leq \frac{1}{b\left(x^{*}, y^{*}\right)}\left(\frac{p d_{b}\left(u^{*}, w^{*}\right)+q d_{b}\left(u^{*}, u^{*}\right)+r d_{b}\left(w^{*}, w^{*}\right)+s \beta d_{b}\left(u^{*}, w^{*}\right) d_{b}\left(w^{*}, u^{*}\right)}{\lambda b\left(u^{*}, u^{*}\right)+\beta\left(d_{b}\left(u^{*}, w^{*}\right)+d_{b}\left(w^{*}, u^{*}\right)\right)}\right) \\
& \leq\left(\frac{p d_{b}\left(u^{*}, w^{*}\right)+s \beta d_{b}\left(u^{*}, w^{*}\right) d_{b}\left(w^{*}, u^{*}\right)}{\lambda b\left(u^{*}, u^{*}\right)+\beta\left(d_{b}\left(u^{*}, w^{*}\right)+d_{b}\left(w^{*}, u^{*}\right)\right)}\right)=\left(\frac{p d_{b}\left(u^{*}, w^{*}\right)+s \beta d_{b}\left(u^{*}, w^{*}\right) d_{b}\left(w^{*}, u^{*}\right)}{\lambda b\left(u^{*}, u^{*}\right)+\beta\left(d_{b}\left(u^{*}, w^{*}\right)+d_{b}\left(w^{*}, u^{*}\right)\right)}\right) \\
& =\frac{p d_{b}\left(u^{*}, w^{*}\right)}{\lambda}+s d_{b}\left(w^{*}, u^{*}\right) .
\end{aligned}
$$

Thus, we get

$$
\left(1-\frac{p}{\lambda}-s\right) d_{b}\left(w^{*}, u^{*}\right) \leq 0
$$

Furthermore, we have to show that $u^{*}$ is a common unique fixed point of $f$ and $g$.

From (1) and (7), we have

Since $(p / 1-s)<\lambda$, we have $1-(p / \lambda)-s>0$ and thus we obtain $d_{b}\left(u^{*}, w^{*}\right)=0$; hence, $u^{*}=w^{*}$.

$$
\begin{aligned}
d_{b}\left(u^{*}, f u^{*}\right) & =d_{b}\left(f x^{*}, f u^{*}\right) \leq \\
& \leq \frac{1}{b\left(x^{*}, u^{*}\right)}\left(\frac{p d_{b}\left(g x^{*}, g u^{*}\right)+q d_{b}\left(g x^{*}, f x^{*}\right)+r d_{b}\left(g u^{*}, f u^{*}\right)+s \beta d_{b}\left(f x^{*}, g u^{*}\right) d_{b}\left(f u^{*}, g x^{*}\right)}{\lambda b\left(f x^{*}, g x^{*}\right)+\beta\left(d_{b}\left(f x^{*}, g u^{*}\right)+d_{b}\left(f u^{*}, g x^{*}\right)\right)}\right) \\
& \leq \frac{p d_{b}\left(g x^{*}, g u^{*}\right)+q d_{b}\left(g x^{*}, f x^{*}\right)+r d_{b}\left(g u^{*}, f u^{*}\right)+s \beta d_{b}\left(f x^{*}, g u^{*}\right) d_{b}\left(f u^{*}, g x^{*}\right)}{\lambda b\left(f x^{*}, g x^{*}\right)+\beta\left(d_{b}\left(f x^{*}, g u^{*}\right)+d_{b}\left(f u^{*}, g x^{*}\right)\right)} .
\end{aligned}
$$

Since $f$ and $g$ are weakly compatible and $f x^{*}=$ $g x^{*}=u^{*}$, so we have $g f x^{*}=f g x^{*}=g u^{*}=f u^{*}$. Thus, from (16) we have

$$
\begin{aligned}
d_{b}\left(u^{*}, f u^{*}\right) & =d_{b}\left(f x^{*}, f u^{*}\right) \\
& \leq \frac{p d_{b}\left(u^{*}, g u^{*}\right)+q d_{b}\left(u^{*}, u^{*}\right)+r d_{b}\left(g u^{*}, f u^{*}\right)+s \beta d_{b}\left(u^{*}, g u^{*}\right) d_{b}\left(f u^{*}, u^{*}\right)}{\lambda b\left(u^{*}, u^{*}\right)+\beta\left(d_{b}\left(u^{*}, g u^{*}\right)+d_{b}\left(f u^{*}, u^{*}\right)\right)} \\
& =\frac{p d_{b}\left(u^{*}, f u^{*}\right)+q d_{b}\left(u^{*}, u^{*}\right)+r d_{b}\left(f u^{*}, f u^{*}\right)+s \beta d_{b}\left(u^{*}, f u^{*}\right) d_{b}\left(f u^{*}, u^{*}\right)}{\lambda b\left(u^{*}, u^{*}\right)+\beta\left(d_{b}\left(u^{*}, g u^{*}\right)+d_{b}\left(f u^{*}, u^{*}\right)\right.} \\
& =\frac{p d_{b}\left(u^{*}, f u^{*}\right)+s \beta d_{b}\left(u^{*}, f u^{*}\right) d_{b}\left(f u^{*}, u^{*}\right)}{\lambda b\left(u^{*}, u^{*}\right)+\beta\left(d_{b}\left(u^{*}, g u^{*}\right)+d_{b}\left(f u^{*}, u^{*}\right)\right.} \\
& \leq \frac{p d_{b}\left(u^{*}, f u^{*}\right)}{\lambda b\left(u^{*}, u^{*}\right)}+s d_{b}\left(u^{*}, f u^{*}\right) \leq \frac{p d_{b}\left(u^{*}, f u^{*}\right)}{\lambda}+s d_{b}\left(u^{*}, f u^{*}\right) .
\end{aligned}
$$


Thus, we get

$$
\left(1-\frac{p}{\lambda}-s\right) d_{b}\left(u^{*}, f u^{*}\right) \leq 0 .
$$

Since $(p / 1-s)<\lambda$, we have $1-(p / \lambda)-s>0$ and thus we obtain $d_{b}\left(u^{*}, f u^{*}\right)=0$; hence, $f u^{*}=u^{*}$. This implies that $u^{*}=f u^{*}=g u^{*}$.
Hence, $u^{*}$ is a common fixed point of $f$ and $g$.

Uniqueness of the common fixed point of $f$ and $g$ are shown as follows.

Suppose $w^{*}$ is another common fixed point of $f$ and $g$,

$$
\begin{aligned}
d_{b}\left(u^{*}, w^{*}\right) & =d_{b}\left(f u^{*}, f w^{*}\right) \\
& \leq \frac{1}{b\left(u^{*}, w^{*}\right)}\left(\frac{p d_{b}\left(g u^{*}, g w^{*}\right)+b q\left(g u^{*}, f u^{*}\right)+r d_{b}\left(g w^{*}, f w^{*}\right)+s \beta d_{b}\left(f u^{*}, g w^{*}\right) d_{b}\left(f w^{*}, g u^{*}\right)}{\lambda b\left(f u^{*}, g u^{*}\right)+\beta\left(d_{b}\left(f u^{*}, g w^{*}\right)+d_{b}\left(f w^{*}, g u^{*}\right)\right)}\right) \\
& \leq\left(\frac{p d_{b}\left(u^{*}, w^{*}\right)+b q\left(u^{*}, u^{*}\right)+r d_{b}\left(w^{*}, w^{*}\right)+s \beta d_{b}\left(u^{*}, w^{*}\right) d_{b}\left(w^{*}, u^{*}\right)}{\lambda b\left(u^{*}, u^{*}\right)+\beta\left(d_{b}\left(u^{*}, w^{*}\right)+d_{b}\left(w^{*}, u^{*}\right)\right)}\right) \\
& =\left(\frac{p d_{b}\left(u^{*}, w^{*}\right)+s \beta d_{b}\left(u^{*}, w^{*}\right) d_{b}\left(w^{*}, u^{*}\right)}{\lambda b\left(u^{*}, u^{*}\right)+\beta\left(d_{b}\left(u^{*}, w^{*}\right)+d_{b}\left(w^{*}, u^{*}\right)\right)}\right) \\
& \leq \frac{p d_{b}\left(u^{*}, w^{*}\right)}{\lambda}+s d_{b}\left(u^{*}, w^{*}\right) .
\end{aligned}
$$

Thus, we get

$$
\left(1-\frac{p}{\lambda}-s\right) d_{b}\left(u^{*}, w^{*}\right) \leq 0 .
$$

Since $(p / 1-s)<\lambda$, we have $1-(p / \lambda)-s>0$ and thus we obtain $d_{b}\left(u^{*}, w^{*}\right)=0$; hence, $u^{*}=w^{*}$.

Hence, $f$ and $g$ have a unique common fixed point in $X$.
By taking $g x=x$ for all $x \in X$ in Theorem 1 , we obtain the following.

Corollary 1 . Let $\left(X, d_{b}\right)$ be a complete extended rectangular $b$-metric space, and let $f: X \longrightarrow X$ be self-mappings such that

$$
b(x, y) d_{b}(f x, f y) \leq \frac{p d_{b}(g x, y)+q d_{b}(x, f x)+r(y, f y)+s \beta d_{b}(f x, y) d_{b}(f y, x)}{\lambda b(f x, x)+\beta\left(d_{b}(f x, y)+d_{b}(f y, x)\right)}
$$

holds for all $x, y \in X, \quad \beta>0, \quad 0<s<1, \quad 0<p+q+$ $r<\lambda, \quad(p / 1-s)<\lambda, \quad$ and $\quad \lim _{n, m \rightarrow \infty} b\left(y_{n}, y_{m}\right)<(\lambda-$ $r / p+q)$, then $f$ has a unique fixed point in $X$.

Example 3. Let $A=[(2 / 3), 1]$ and $B=[0,(1 / 2)]$. Let $X=A \cup B$, and define $b(x, y)=2(x+y)+1$ for all $x, y \in X$, $\lambda=.9$, and define $d_{b}: X \times X \longrightarrow[0, \infty)$ as follows:

$$
d_{b}(x, y)= \begin{cases}0, & \text { if } x=y, \\ \frac{1}{2}, & \text { if } x, y \in A \\ 1, & \text { if } x, y \in B \\ (x-y)^{2}, & \text { otherwise. }\end{cases}
$$

It is easy that $\left(X, d_{b}\right)$ is an extended rectangular space with $b(x, y)=0.2(x+y)+1$.

Let $f(x)=x^{4} / 4$ and $g(x)=x^{2} / 2$, for all $x \in X$. It is clear that $f X \subseteq g X$.

By using $y_{n}=f\left(x_{n}\right)=g\left(x_{n+1}\right), \quad f(x)=x^{4} / 2^{4}$, and $g(x)=x^{2} / 2^{2}$, we have

$$
\begin{aligned}
& y_{0}=f\left(x_{0}\right)=\frac{x^{4}}{2^{4}}=g\left(x_{1}\right) \text { thus } x_{1}=\frac{x^{2}}{2}, \\
& y_{1}=f\left(x_{1}\right)=f\left(\frac{x^{2}}{2}\right)=\frac{x^{8}}{2^{8}}=g\left(x_{2}\right) \text { thus } x_{2}=\frac{x^{4}}{2^{3}}, \\
& y_{2}=f\left(x_{2}\right)=f\left(\frac{x^{4}}{2^{3}}\right)=\frac{x^{16}}{2^{16}}=g\left(x_{2}\right) \text { thus } x_{3}=\frac{x^{8}}{2^{7}} .
\end{aligned}
$$

Generally, we have

$$
\begin{aligned}
y_{n} & =\frac{x^{2^{n+2}}}{2^{n+2}}, \\
x_{n} & =\frac{x^{2^{n}}}{2^{2^{n}-1}}, \\
\lim _{n, m \longrightarrow \infty} b\left(y_{n}, y_{m}\right) & =\lim _{n, m \longrightarrow \infty} 2+y_{n}+y_{m} \\
& =\lim _{n, m \longrightarrow \infty} 1+\frac{x^{2^{n+2}}}{2^{n+2}}+\frac{x^{2^{m+2}}}{2^{m+2}} \\
& =1<\frac{\lambda-r}{p+q}=\frac{0.9-0.4}{0.1+0.3}=\frac{0.5}{0.4}=1.25 .
\end{aligned}
$$


Since $x \in[0,1]$, the sequence $\left\{y_{n}\right\}=\left\{x^{2^{n+2}} / 2^{n+2}\right\}$ is convergent to 0 . Indeed, $\lim _{n \longrightarrow \infty} d_{b}\left(x_{n}, 0\right)=0$.
(1) For $x, y \in A=[(2 / 3), 1]$ or $x, y \in B=[0,(1 / 2)]$, it is clear that $b(x, y) d_{b}(f x, f y)=0$

(2) For $x \in A=[(2 / 3), 1]$ and $y \in B=[0,(1 / 2)]$

$$
\begin{aligned}
& \text { For } x \in A=\left[\frac{2}{3}, 1\right] \text { then } f(x)=\frac{x^{4}}{4} \in\left[\frac{16}{324}, \frac{1}{4}\right] \subseteq B=\left[0, \frac{1}{2}\right] \\
& \text { For } x \in A=\left[\frac{2}{3}, 1\right] \text { then } g(x)=\frac{x^{2}}{2} \in\left[\frac{4}{18}, \frac{1}{2}\right] \subseteq B=\left[0, \frac{1}{2}\right] \\
& \text { For } y \in B=\left[0, \frac{1}{2}\right] \text { then } f(y)=\frac{y^{4}}{4} \in\left[0, \frac{1}{32}\right] \subseteq B=\left[0, \frac{1}{2}\right] \\
& \text { For } y \in B=\left[0, \frac{1}{2}\right] \text { then } g(y)=\frac{y^{2}}{2} \in\left[0, \frac{1}{4}\right] \subseteq B=\left[0, \frac{1}{2}\right] \\
& \begin{array}{l}
p d_{b}(g x, g y)+q d_{b}(g x, f x)+r(g y, f y)+s \beta d_{b}(f x, g y) d_{b}(f y, g x) \\
\lambda b(f x, g x)+\beta\left(d_{b}(f x, g y)+d_{b}(f y, g x)\right)
\end{array} \\
& =\frac{0.1 d_{b}\left(x^{2} / 2, y^{2} / 2\right)+0.3 d_{b}\left(x^{2} / 2, x^{4} / 4\right)+0.4\left(y^{2} / 2, y^{4} / 4\right)+0.5(0.1) d_{b}\left(x^{4} / 4, y^{2} / 2\right) d_{b}\left(y^{4} / 4, x^{2} / 2\right)}{0.9 b\left(x^{4} / 4, x^{2} / 2\right)+0.1\left(d_{b}\left(x^{4} / 4, y^{2} / 2\right)+d_{b}\left(y^{4} / 4, x^{2} / 2\right)\right)} \\
& =\frac{0.1+0.3+0.4+0.5(0.1)}{0.9(0.2)\left(x^{4} / 4, x^{2} / 2+1\right)+0.1(1+1)}=\frac{0.85}{0.18\left(x^{4} / 4, x^{2} / 2+1\right)+0.2}, \\
& b(x, y) d_{b}(f x, f y)=\left(0.2\left(x^{4} / 4, x^{2} / 2\right)+1\right) d_{b}\left(x^{4} / 4, y^{4} / 4\right)=\left(0.2\left(x^{4} / 4, x^{2} / 2\right)+1\right) \leq \frac{18\left(x^{4} / 4, x^{2} / 2+1\right)+0.1}{0.1}
\end{aligned}
$$

The largest value of $b(x, y) d_{b}(f x, f y)$ occurs if $x=1$ and $y=1 / 2$. So, we have

$$
\begin{aligned}
b(x, y) d_{b}(f x, f y) & =\left(0.2\left(\frac{x^{4}}{4}+\frac{x^{2}}{2}\right)+1\right)=\left(0.2\left(\frac{1}{4}+\frac{1}{8}\right)+1\right)=1.275 \\
& \leq \frac{p d_{b}(g x, g y)+q d_{b}(g x, f x)+r(g y, f y)+s \beta d_{b}(f x, g y) d_{b}(f y, g x)}{\lambda b(f x, g x)+\beta\left(d_{b}(f x, g y)+d_{b}(f y, g x)\right)} \\
& \leq \frac{0.85}{0.18(11 / 8)+0.2}=1.899 .
\end{aligned}
$$

(3) For $x \in B=[0,(1 / 2)]$ and $y \in A=[(2 / 3), 1]$,

$$
\begin{aligned}
& \text { For } x \in B=\left[0, \frac{1}{2}\right] \text { then } f(x)=\frac{x^{4}}{4} \in\left[0, \frac{1}{32}\right] \subseteq B=\left[0, \frac{1}{2}\right], \\
& \text { For } x \in B=\left[0, \frac{1}{2}\right] \text { then } g(x)=\frac{x^{2}}{2} \in\left[\frac{0,1}{8}\right] \subseteq B=\left[0, \frac{1}{2}\right], \\
& \text { For } y \in A=\left[\frac{2}{3}, 1\right] \text { then } f(y)=\frac{y^{4}}{4} \in\left[\frac{16}{324}, \frac{1}{4}\right] \subseteq B=\left[0, \frac{1}{2}\right] \text {, } \\
& \text { For } y \in A=\left[\frac{2}{3}, 1\right] \text { then } g(y)=\frac{y^{2}}{2} \in\left[\frac{4}{18}, \frac{1}{2}\right] \subseteq B=\left[0, \frac{1}{2}\right],
\end{aligned}
$$




$$
\begin{aligned}
& \frac{p d_{b}(g x, g y)+q d_{b}(g x, f x)+r(g y, f y)+s \beta d_{b}(f x, g y) d_{b}(f y, g x)}{\lambda b(f x, g x)+\beta\left(d_{b}(f x, g y)+d_{b}(f y, g x)\right)} \\
& =\frac{0.1 d_{b}\left(x^{2} / 2, y^{2} / 2\right)+0.3 d_{b}\left(x^{2} / 2, x^{4} / 4\right)+0.4\left(y^{2} / 2, y^{4} / 4\right)+0.5(0.1) d_{b}\left(x^{4} / 4, y^{2} / 2\right) d_{b}\left(y^{4} / 4, x^{2} / 2\right)}{0.9 b\left(x^{4} / 4, x^{2} / 2\right)+0.1\left(d_{b}\left(x^{4} / 4, y^{2} / 2\right)+d_{b}\left(y^{4} / 4, x^{2} / 2\right)\right)} \\
& =\frac{0.1+0.3+0.4+0.5(0.1)}{0.9(0.2)\left(x^{4} / 4+x^{2} / 2+1\right)+0.1(1+1)}=\frac{0.85}{0.18\left(x^{4} / 4+x^{2} / 2+1\right)+0.2}, \\
& b(x, y) d_{b}(f x, f y)=\left(0.2\left(\frac{x^{4}}{4}+\frac{x^{2}}{2}\right)+1\right) d_{b}\left(\frac{x^{4}}{4}, \frac{y^{4}}{4}\right)=\left(0.2\left(\frac{x^{4}}{4}+\frac{x^{2}}{2}\right)+1\right) \leq \frac{0.85}{0.18\left(x^{4} / 4+x^{2} / 2+1\right)+0.2}
\end{aligned}
$$

The largest value of $b(x, y) d_{b}(f x, f y)$ occurs if $x=1 / 2$ and $y=1$. So, we have

$$
\begin{aligned}
b(x, y) d_{b}(f x, f y) & =\left(0.2\left(\frac{x^{4}}{4}+\frac{x^{2}}{2}\right)+1\right) d_{b}\left(\frac{x^{4}}{4}, \frac{y^{4}}{4}\right)=\left(0.2\left(\frac{1}{4}+\frac{1}{8}\right)+1\right)=1.275 \\
& \leq \frac{p d_{b}(g x, g y)+q d_{b}(g x, f x)+r(g y, f y)+s \beta d_{b}(f x, g y) d_{b}(f y, g x)}{\lambda b(f x, g x)+\beta\left(d_{b}(f x, g y)+d_{b}(f y, g x)\right)} \\
& \leq \frac{0.85}{0.18(11 / 8)+0.2}=1.899 .
\end{aligned}
$$

Hence, we get that condition

$$
b(x, y) d_{b}(f x, f y) \leq \frac{p d_{b}(g x, g y)+q d_{b}(g x, f x)+r(g y, f y)+s \beta d_{b}(f x, g y) d_{b}(f y, g x)}{\lambda b(f x, g x)+\beta\left(d_{b}(f x, g y)+d_{b}(f y, g x)\right)}
$$

is hold.

Hence, from Theorem 1 , we have $x=0$ is a unique common fixed point of $f$ and $g$.

Theorem 2. Let $\left(X, d_{b}\right)$ be a complete extended rectangular $b$-metric space, and let $f, g: X \longrightarrow X$ be self-mappings such that $f(X) \subseteq g(X)$ and $g(X)$ closed. If the conditions

$$
\begin{aligned}
b(x, y) d_{b}(f x, f y) \leq & p d_{b}(g x, g y)+\frac{q}{b(f x, g x)} d_{b}(g x, f x) \\
& +r d_{b}(g y, f y) \\
& +\frac{s d_{b}(f x, g y) d_{b}(f y, g x)}{1+d_{b}(f x, g y)+d_{b}(f y, g x)},
\end{aligned}
$$

hold for all $x, y \in X$, where $0<q, r<1,0<p+s<1, q, r<$ $1,0<p+s<1,0<(p+q / 1-r)<1$, and $\lim _{n, m \rightarrow \infty} b\left(x_{n}\right.$, $\left.y_{m}\right)<(1-r / p+q)$, and then $f$ and $g$ have a unique coincidence in $X$.

Moreover, if $f$ and $g$ have weakly compatible property, then $f$ and $g$ have a unique fixed point.

Proof. Let taking $x_{0} \in X$ as initial point define sequence $\left\{x_{n}\right\}$ and $\left\{y_{n}\right\}$ in $X$ such that $y_{n}=$ $f\left(x_{n}\right)=g\left(x_{n+1}\right), n=0,1,2, \ldots$ Suppose that $y_{n} \neq y_{n+1}$, $n=0,1,2, \ldots$, because if $y_{n}=y_{n+1}$ for some $n$, then $g\left(x_{n+1}\right)=y_{n}=y_{n+1}=f\left(x_{n+1}\right)$, so $f$ and $g$ have a coincidence point.

Consider from (1) and (32), we have 


$$
\begin{aligned}
b\left(x_{n}, x_{n+1}\right) d_{b}\left(y_{n}, y_{n+1}\right)= & b\left(x_{n}, x_{n+1}\right) d_{b}\left(f x_{n}, f x_{n+1}\right) \\
\leq & p d_{b}\left(g x_{n}, g x_{n+1}\right)+\frac{q}{b\left(f x_{n}, g x_{n}\right)} d_{b}\left(g x_{n}, f x_{n}\right)+r d_{b}\left(g x_{n+1}, f x_{n+1}\right) \\
& +\frac{s d_{b}\left(f x_{n}, g x_{n+1}\right) d_{b}\left(f x_{n+1}, g x_{n}\right)}{1+d_{b}\left(f x_{n}, g x_{n+1}\right)+d_{b}\left(f x_{n+1}, g x_{n}\right)} \\
= & p d_{b}\left(y_{n-1}, y_{n}\right)+\frac{q}{b\left(y_{n}, y_{n-1}\right)} d_{b}\left(y_{n-1}, y_{n}\right)+r d_{b}\left(y_{n}, y_{n+1}\right)+\frac{s d_{b}\left(y_{n}, y_{n}\right) d_{b}\left(y_{n+1}, y_{n-1}\right)}{1+d_{b}\left(y_{n}, y_{n}\right)+d_{b}\left(y_{n+1}, y_{n-1}\right)} \\
\leq & (p+q) d_{b}\left(y_{n-1}, y_{n}\right)+r d_{b}\left(y_{n}, y_{n+1}\right) .
\end{aligned}
$$

Since $b\left(x_{n}, x_{n+1}\right) \geq 1$, we get

$$
\begin{aligned}
d_{b}\left(y_{n}, y_{n+1}\right) & \leq \frac{p+q}{b\left(x_{n}, x_{n+1}\right)-r} d_{b}\left(y_{n-1}, y_{n}\right) \\
& \leq \frac{p+q}{1-r} d_{b}\left(y_{n-1}, y_{n}\right) .
\end{aligned}
$$

So, we get

$$
d_{b}\left(y_{n}, y_{n+1}\right) \leq \beta d_{b}\left(y_{n-1}, y_{n}\right)
$$

where $\beta=p+q / 1-r$. Since as known $0<\beta=(p+q / 1-$ $r)<1$ and $\lim _{n, m \rightarrow \infty} b\left(y_{n}, y_{m}\right)<(1 / \beta)$, then by using Lemma 2, we get $\left\{y_{n}\right\}$ is a Cauchy sequence in $g X$.
Since $X$ is complete, then there exists $u^{*} \in X$ such that

$$
d_{b}\left(y_{n}, u^{*}\right) \longrightarrow 0 \text {. }
$$

Since $y_{n}=g\left(x_{n+1}\right) \subseteq X$ and $g X$ closed, then $u^{*} \in g X$ and $u^{*}=g\left(x^{*}\right)$ for some $x^{*} \in X$.

Hence, for $n, m \longrightarrow \infty$, in (16) we get $d_{b}\left(y_{n}, y_{m}\right) \longrightarrow 0$; thus, $\left\{y_{n}\right\}$ is the Cauchy sequence in $g X$.

Furthermore, we will show that $f$ and $g$ have a point coincidence, as follows.

From (1) and (32), we have

$$
\begin{aligned}
d_{b}\left(f x^{*}, g x^{*}\right) \leq & b\left(f x^{*}, g x^{*}\right)\left[d_{b}\left(f x^{*}, y_{n}\right)+d_{b}\left(y_{n}, y_{n+1}\right)+d_{b}\left(y_{n+1}, g x^{*}\right)\right. \\
= & b\left(x^{*}, x^{*}\right)\left[d_{b}\left(f x^{*}, f x_{n}\right)+d_{b}\left(y_{n}, y_{n+1}\right)+d_{b}\left(y_{n+1}, u^{*}\right)\right] \\
\leq & b\left(f x^{*}, g x^{*}\right)\left[\frac { 1 } { b ( x ^ { * } , x _ { n } ) } \left(p d_{b}\left(g x^{*}, g x_{n}\right)+\frac{q}{b\left(f x^{*}, g x^{*}\right)} d_{b}\left(g x^{*}, f x^{*}\right)+r d_{b}\left(g x_{n}, f x_{n}\right)\right.\right. \\
& \left.\left.+\frac{s d_{b}\left(f x^{*}, g x_{n}\right) d_{b}\left(f x_{n}, g x^{*}\right)}{1+d_{b}\left(f x^{*}, g x_{n}\right)+d_{b}\left(f x_{n}, g x^{*}\right)}\right)+d_{b}\left(y_{n}, y_{n+1}\right)+d_{b}\left(y_{n+1}, u^{*}\right)\right] \\
\leq & b\left(f x^{*}, g x^{*}\right)\left[\frac { 1 } { b ( x ^ { * } , x _ { n } ) } \left(p d_{b}\left(u^{*}, y_{n-1}\right)+\frac{q}{b\left(f x^{*}, g x^{*}\right)} d_{b}\left(u^{*}, f x^{*}\right)+r d_{b}\left(y_{n-1}, y_{n}\right)\right.\right. \\
& \left.\left.+\frac{s d_{b}\left(f x^{*}, y_{n-1}\right) d_{b}\left(y_{n}, u^{*}\right)}{1+d_{b}\left(f x^{*}, y_{n-1}\right)+d_{b}\left(y_{n}, u^{*}\right)}\right)+d_{b}\left(y_{n}, y_{n+1}\right)+d_{b}\left(y_{n+1}, u^{*}\right)\right] \\
\leq & b\left(f x^{*}, g x^{*}\right)\left[\frac{1}{b\left(x^{*}, x_{n}\right)}\left(p d_{b}\left(u^{*}, y_{n-1}\right)+\frac{q}{b\left(f x^{*}, g x^{*}\right)} d_{b}\left(u^{*}, f x^{*}\right)+r d_{b}\left(y_{n-1}, y_{n}\right)+s d_{b}\left(y_{n}, u^{*}\right)\right)\right. \\
& \left.+d_{b}\left(y_{n}, y_{n+1}\right)+d_{b}\left(y_{n+1}, u^{*}\right)\right] .
\end{aligned}
$$

By using (36) and (37), if $n \longrightarrow \infty$, then we get

$$
\begin{aligned}
d_{b}\left(f x^{*}, g x^{*}\right) & \leq \frac{q d_{b}\left(u^{*}, f x^{*}\right)}{b\left(x^{*}, x_{n}\right)}=\frac{q d_{b}\left(g x^{*}, f x^{*}\right)}{b\left(x^{*}, x_{n}\right)} \\
& \leq q d_{b}\left(g x^{*}, f x^{*}\right) .
\end{aligned}
$$

Since $0<q<1$, this implies that $d_{b}\left(f x^{*}, g x^{*}\right)=0$, so then we get $f x^{*}=g x^{*}=u^{*}$. Hence, $u^{*}$ is a coincidence point of $f$ and $g$.

To show uniqueness of the coincidence point of $f$ and $g$, we suppose there exists $w^{*} \in X$ such that $f y^{*}=g y^{*}=w^{*}$ : 


$$
\begin{aligned}
d_{b}\left(u^{*}, w^{*}\right) & =d_{b}\left(f x^{*}, f y^{*}\right) \\
& \leq \frac{1}{b\left(x^{*}, y^{*}\right)}\left[p d_{b}\left(g x^{*}, g y^{*}\right)+\frac{q}{b\left(f x^{*}, g x^{*}\right)} d_{b}\left(g x^{*}, f x^{*}\right)+r d_{b}\left(g y^{*}, f y^{*}\right)+\frac{s d_{b}\left(f x^{*}, g y^{*}\right) d_{b}\left(f y^{*}, g x^{*}\right)}{1+d_{b}\left(f x^{*}, g y^{*}\right)+d_{b}\left(f y^{*}, g x^{*}\right)}\right] \\
& \leq p d_{b}\left(u^{*}, w^{*}\right)+\frac{q}{b\left(u^{*}, u^{*}\right)} d_{b}\left(u^{*}, u^{*}\right)+r d_{b}\left(w^{*}, w^{*}\right)+\frac{s d_{b}\left(u^{*}, w^{*}\right) d_{b}\left(w^{*}, u^{*}\right)}{1+d_{b}\left(u^{*}, w^{*}\right)+d_{b}\left(w^{*}, g u^{*}\right)} \\
& \leq p d_{b}\left(u^{*}, w^{*}\right)+\frac{q}{b\left(u^{*}, u^{*}\right)} d_{b}\left(u^{*}, u^{*}\right)+r d_{b}\left(w^{*}, w^{*}\right)+s d_{b}\left(u^{*}, w^{*}\right) \\
& \leq p d_{b}\left(u^{*}, w^{*}\right)+s d_{b}\left(u^{*}, w^{*}\right)=(p+s) d_{b}\left(u^{*}, w^{*}\right) .
\end{aligned}
$$

Thus, we get

$$
(1-p-s) d_{b}\left(u^{*}, w^{*}\right) \leq 0
$$

Since $0<p+s<1$, then we get $d_{b}\left(u^{*}, w^{*}\right)=0$; hence, $u^{*}=w^{*}$.

Next, we have to show that $u^{*}$ is a common fixed point of $f$ and $g$. Consider that

$$
\begin{aligned}
d_{b}\left(u^{*}, f u^{*}\right) & =d_{b}\left(f x^{*}, f u^{*}\right) \\
& \leq \frac{1}{b\left(x^{*}, u^{*}\right)}\left[p d_{b}\left(g x^{*}, g u^{*}\right)+\frac{q}{b\left(f x^{*}, g x^{*}\right)} d_{b}\left(g x^{*}, f x^{*}\right)+r d_{b}\left(g u^{*}, f u^{*}\right)+\frac{s d_{b}\left(f x^{*}, g u^{*}\right) d_{b}\left(f u^{*}, g x^{*}\right)}{1+d_{b}\left(f x^{*}, g u^{*}\right)+d_{b}\left(f u^{*}, g x^{*}\right)}\right] .
\end{aligned}
$$

Since $f$ and $g$ are weakly compatible and $f x^{*}=$ $g x^{*}=u^{*}$, we have $g f x^{*}=f g x^{*}=g u^{*}=f u^{*}$. Thus, from (41) we get

$$
\begin{aligned}
d_{b}\left(u^{*}, f u^{*}\right) & =d_{b}\left(f x^{*}, f u^{*}\right) \\
& \leq \frac{1}{b\left(x^{*}, u^{*}\right)}\left[p d_{b}\left(g x^{*}, g u^{*}\right)+\frac{q}{b\left(f x^{*}, g x^{*}\right)} d_{b}\left(g x^{*}, f x^{*}\right)+r d_{b}\left(g u^{*}, f u^{*}\right)+\frac{s d_{b}\left(f x^{*}, g u^{*}\right) d_{b}\left(f u^{*}, g x^{*}\right)}{1+d_{b}\left(f x^{*}, g u^{*}\right)+d_{b}\left(f u^{*}, g x^{*}\right)}\right] \\
& \leq \frac{1}{b\left(x^{*}, u^{*}\right)}\left[p d_{b}\left(u^{*}, f u^{*}\right)+\frac{q}{b\left(u^{*}, u^{*}\right)} d_{b}\left(u^{*}, u^{*}\right)+r d_{b}\left(f u^{*}, f u^{*}\right)+\frac{s d_{b}\left(u^{*}, f u^{*}\right) d_{b}\left(f u^{*}, u^{*}\right)}{1+d_{b}\left(u^{*}, f u^{*}\right)+d_{b}\left(f u^{*}, u^{*}\right)}\right] \\
& \leq \frac{p}{b\left(x^{*}, u^{*}\right)} d_{b}\left(u^{*}, f u^{*}\right)+s d_{b}\left(u^{*}, f u^{*}\right) \leq p d_{b}\left(u^{*}, f u^{*}\right)+s d_{b}\left(u^{*}, f u^{*}\right) \\
& \leq(p+s) d_{b}\left(u^{*}, f u^{*}\right) .
\end{aligned}
$$

Thus, we obtain $(1-p-s) d_{b}\left(u^{*}, f u^{*}\right) \leq 0$.

Since $p+s<1$, we obtain that $d_{b}\left(u^{*}, f u^{*}\right)=0$. This implies that $u^{*}=f u^{*}=g u^{*}$.

Hence, $u^{*}$ is a common fixed point of $f$ and $g$.
Uniqueness of the common fixed point of $f$ and $g$ are shown as follows.

Suppose $w^{*}$ is another common fixed point of $f$ and $g$, that is, $w^{*}=f w^{*}=g w^{*}$. 
$d_{b}\left(u^{*}, w^{*}\right)=d_{b}\left(f u^{*}, f w^{*}\right)$

$$
\begin{aligned}
& \leq \frac{1}{b\left(u^{*}, w^{*}\right)}\left[p d_{b}\left(g u^{*}, g w^{*}\right)+\frac{q}{b\left(f u^{*}, g u^{*}\right)} d_{b}\left(g u^{*}, f u^{*}\right)+r d_{b}\left(g w^{*}, f w^{*}\right)+\frac{s d_{b}\left(f u^{*}, g w^{*}\right) d_{b}\left(f w^{*}, f u^{*}\right)}{1+d_{b}\left(f u^{*}, g w^{*}\right)+d_{b}\left(f w^{*}, f u^{*}\right)}\right] \\
& \leq \frac{1}{b\left(u^{*}, w^{*}\right)}\left[p d_{b}\left(u^{*}, w^{*}\right)+\frac{q}{b\left(u^{*}, u^{*}\right)} d_{b}\left(u^{*}, u^{*}\right)+r d_{b}\left(w^{*}, w^{*}\right)+\frac{s d_{b}\left(u^{*}, w^{*}\right) d_{b}\left(w^{*}, u^{*}\right)}{1+d_{b}\left(u^{*}, w^{*}\right)+d_{b}\left(w^{*}, u^{*}\right)}\right] \\
& =\frac{p d_{b}\left(u^{*}, w^{*}\right)}{b\left(u^{*}, w^{*}\right)}+s d_{b}\left(u^{*}, w^{*}\right) \leq(p+s) d_{b}\left(u^{*}, w^{*}\right) .
\end{aligned}
$$

Since $0<p+s<1$, we obtain that $d_{b}\left(u^{*}, w^{*}\right)=0$, and it implies that $u^{*}=w^{*}$.

Hence, $f$ and $g$ have a unique common fixed point in $X$.

By taking $g x=x$ in Theorem 2 we obtain

Corollary 2. Let $\left(X, d_{b}\right)$ be a complete extended rectangular $b$-metric space, and let $f: X \longrightarrow X$ be a self-mapping such that

$$
\begin{aligned}
b(x, y) d_{b}(f x, f y) \leq & p d_{b}(x, y)+\frac{q}{b(f x, x)} d_{b}(x, f x) \\
& +r d_{b}(y, f y) \\
& +\frac{s d_{b}(f x, y) d_{b}(f y, x)}{1+d_{b}(f x, y)+d_{b}(f y, x)}
\end{aligned}
$$

holds for all $x, y \in X$, where $0<q, r<1,0<p+s<1$, $q, r<1,0<p+s<1, \quad 0<(p+q / 1-r)<1 \quad$ and $\lim _{n, m \rightarrow \infty} b\left(x_{n}, y_{m}\right)<(1-r / p+q)$, and then $f$ has $a$ unique fixed point in $X$.

Example 4. We use $f(x)=x^{4} / 4, g(x)=x^{2} / 2$ and $d_{b}(x, y)$ in Example 3, and taking $b(x, y)=0.1(x+y)+1$, $p=0.2, q=0.3, r=0.45$, and $s=0.7$. These parameters satisfy for conditions

$$
\begin{gathered}
0<r<1,0<p+s<1,0<\frac{p+q}{1-r}<1, \\
\lim _{n, m \rightarrow \infty} b\left(x_{n}, y_{m}\right)<\frac{1-r}{p+q} .
\end{gathered}
$$

From Example 3 we have

$$
\begin{aligned}
& y_{n}=\frac{x^{2^{n+2}}}{2^{n+2}}, \\
& x_{n}=\frac{x^{2^{n}}}{2^{2^{n}-1}} .
\end{aligned}
$$

Thus, we have

$$
\begin{aligned}
\lim _{n, m \rightarrow \infty} b\left(y_{n}, y_{m}\right) & =\lim _{n, m \rightarrow \infty} 0.1\left(y_{n}+y_{m}\right)+1 \\
& =\lim _{n, m \longrightarrow \infty} 0.1\left(\frac{x^{2^{n+2}}}{2^{n+2}}+\frac{x^{2^{m+2}}}{2^{m+2}}\right)+1 \\
& =1<\frac{1-r}{p+q}=\frac{1-0.4}{0.2+0.3}=\frac{0.6}{0.5}=1.2 .
\end{aligned}
$$

Since $0 \leq x \leq 1$, the sequence $\left\{y_{n}\right\}=\left\{x^{2^{n+2}} / 2^{n+2}\right\}$ is convergent to 0 . Indeed, $\lim _{n \rightarrow \infty} d_{b}\left(y_{n}, 0\right)=0$.

(1) For $x, y \in A=[(2 / 3), 1]$ or $x, y \in B=[0,(1 / 2)]$, it is clear that $b(x, y) d_{b}(f x, f y)=0$

(2) For $x \in A=[(2 / 3), 1]$ and $y \in B=[0,(1 / 2)]$

$$
\begin{aligned}
& \text { For } x \in A=\left[\frac{2}{3}, 1\right] \text { then } f(x)=\frac{x^{4}}{4} \in\left[\frac{16}{324}, \frac{1}{4}\right] \subseteq B=\left[0, \frac{1}{2}\right] \\
& \text { For } x \in A=\left[\frac{2}{3}, 1\right] \text { then } g(x)=\frac{x^{2}}{2} \in\left[\frac{4}{18}, \frac{1}{2}\right] \subseteq B=\left[0, \frac{1}{2}\right] \\
& \text { For } y \in B=\left[0, \frac{1}{2}\right] \text { then } f(y)=\frac{y^{4}}{4} \in\left[0, \frac{1}{32}\right] \subseteq B=\left[0, \frac{1}{2}\right] \\
& \text { For } y \in B=\left[0, \frac{1}{2}\right] \text { then } g(y)=\frac{y^{2}}{2} \in\left[0, \frac{1}{4}\right] \subseteq B=\left[0, \frac{1}{2}\right]
\end{aligned}
$$




$$
\begin{aligned}
& b(x, y) d_{b}(f x, f y) \\
& \leq 0.2 d_{b}(g x, g y)+\frac{0.3}{b(f x, g x)} d_{b}(g x, f x)+0.45 d_{b}(g y, f y)+\frac{0.7 d_{b}(f x, g y) d_{b}(f y, g x)}{1+d_{b}(f x, g y)+d_{b}(f y, g x)} \\
& =0.2 d_{b}\left(\frac{x^{2}}{2}, \frac{y^{4}}{4}\right)+\frac{0.3}{b\left(x^{4} / 4, x^{2} / 2\right)} d_{b}\left(x^{2} / 2, x^{4} / 4\right)+0.45 d_{b}\left(\frac{y^{2}}{2}, \frac{y^{4}}{4}\right)+\frac{0.7 d_{b}\left(y^{4} / 4, y^{2} / 2\right) d_{b}\left(y^{4} / 4, x^{2} / 2\right)}{1+d_{b}\left(y^{4} / 4, y^{2} / 2\right)+d_{b}\left(y^{4} / 4, x^{2} / 2\right)} \\
& \left.=0.2+\frac{0.3}{0.2\left(x^{4} / 4+x^{2} / 2\right)+1}+0.45+\frac{0.7}{1+1+1}=0.65+\frac{0.3}{0.2\left(x^{4} / 4+x^{2} / 2\right)+1}+\frac{x^{4}}{3}, \frac{y^{4}}{4}\right)=0.2(x+y)+1 \\
& b(x, y) d_{b}(f x, f y)=(0.2(x+y)+1) d_{b}\left(\frac{x^{4}}{4}\right)
\end{aligned}
$$

The largest value of $b(x, y) d_{b}(f x, f y)$ occurs if $x=1$ and $y=1 / 2$. So, we have

$$
\begin{aligned}
b(x, y) d_{b}(f x, f y) & =(0.1(x+y)+1) d_{b}\left(\frac{x^{4}}{4}, \frac{y^{4}}{4}\right) \\
& =0.1(x+y)+1=0.1\left(1+\frac{1}{2}\right)+1=1.1 .
\end{aligned}
$$

And we have

$$
\begin{aligned}
& 2 d_{b}(g x, g y)+\frac{0.3}{b(f x, g x)} d_{b}(g x, f x) \\
& \quad+0.45 d_{b}(g y, f y)+\frac{0.7 d_{b}(f x, g y) d_{b}(f y, g x)}{1+d_{b}(f x, g y)+d_{b}(f y, g x)} \\
& =0.65+\frac{0.3}{0.1\left(\left(x^{4} / 4\right)+\left(x^{2} / 2\right)\right)+1} \\
& \quad+\frac{0.7}{3}==0.65+\frac{0.3}{0.1((1 / 4)+(1 / 2))+1}+\frac{0.7}{3}=1.17
\end{aligned}
$$

So, we get that

$$
\begin{aligned}
b(x, y) d_{b}(f x, f y) \leq & 2 d_{b}(g x, g y)+\frac{0.3}{b(f x, g x)} d_{b}(g x, f x) \\
& +0.45 d_{b}(g y, f y) \\
& +\frac{0.7 d_{b}(f x, g y) d_{b}(f y, g x)}{1+d_{b}(f x, g y)+d_{b}(f y, g x)}
\end{aligned}
$$

(3) For $x \in B=[0,(1 / 2)]$ and $y \in A=[(2 / 3), 1]$

Similarly, we also obtain that

$$
\begin{aligned}
b(x, y) d_{b}(f x, f y) \leq & 2 d_{b}(g x, g y)+\frac{0.3}{b(f x, g x)} d_{b} \\
& \cdot(g x, f x)+0.45 d_{b}(g y, f y) \\
& +\frac{0.7 d_{b}(f x, g y) d_{b}(f y, g x)}{1+d_{b}(f x, g y)+d_{b}(f y, g x)}
\end{aligned}
$$

Hence, we get that the condition

$$
\begin{aligned}
b(x, y) d_{b}(f x, f y) \leq & p d_{b}(x, y)+\frac{q}{b(f x, x)} d_{b}(x, f x) \\
& +r d_{b}(y, f y), \\
& +\frac{s d_{b}(f x, y) d_{b}(f y, x)}{1+d_{b}(f x, y)+d_{b}(f y, x)} .
\end{aligned}
$$

for all $x, y \in X$ holds.

Hence, from Theorem 2, we can conclude that $x=0$ is a unique common fixed point of $f$ and $g$.

\section{Data Availability}

No data were used to support this study.

\section{Conflicts of Interest}

The author declares that there are no conflicts of interest.

\section{Acknowledgments}

The author is grateful to Hasanuddin University for financial support to this work by BMIS Research Project 2017 (no. 3556/UN.4.3.2/LK.23/2017).

\section{References}

[1] S. Czerwik, "Contraction mappings in b-metric spaces," Acta Mathematica et Informatica Universitatis Ostraviensis, vol. 1, no. 1, pp. 5-11, 1993.

[2] S. K. Mohanta, "Coincidence points and common fixed points for expansive type mappings in $b$-metric spaces," Iranian Journal of Mathematical Sciences and Informatics, vol. 11, pp. 101-113, 2016.

[3] N. Hussain, V. Parvaneh, B. A. S. Alamri, and Z. Kadelburg, "F-HR-type contractions on $(\alpha, \eta)$-complete rectangular $b$ metric spaces," The Journal of Nonlinear Sciences and Applications, vol. 10, no. 3, pp. 1030-1043, 2017.

[4] T. Kamran, M. Samreen, and Q. Ul Ain, "A generalization of $b$-metric space and some fixed point theorems," Mathematics, vol. 5, no. 2, p. 19, 2017.

[5] B. Alqahtani, A. Fulga, and E. Karapınar, "Common fixed point results on an extended b-metric space," Journal of Inequalities and Applications, vol. 2018, no. 1, p. 158, 2018. 
[6] R. George, S. Radenović, K. P. Reshma, and S. Shukla, "Rectangular $b$-metric space and contraction principles," Journal of Nonlinear Sciences and Applications, vol. 8, no. 6, pp. 1005-1013, 2015.

[7] Z. Kadelburg and S. Radenović, "Pata-type common fixed point results in $b$-metric and $b$-rectangular metric spaces," Journal of Nonlinear Sciences and Applications, vol. 8, no. 6, pp. 944-954, 2015.

[8] Z. D. Mitrović, "A note on a Banach's fixed point theorem in $b$-rectangular metric space and $b$-metric space," Mathematica Slovaca, vol. 68, no. 5, pp. 1113-1116, 2018.

[9] M. Asim, M. Imdad, and S. Radenovic, "Fixed point results in extended rectangular $b$-metric spaces with an application," University Politehnica of Bucharest Scientific Bulletin-Series A-Applied Mathematics and Physics, vol. 81, no. 2, pp. 43-50, 2019.

[10] Z. Mustafa, V. Parvaneh, M. M. M. Jaradat, and Z. Kadelburg, "Extended rectangular $b$-metric spaces and some fixed point theorems for contractive mappings," Symmetry, vol. 11, no. 4, p. 594, 2019.

[11] N. V. Dung, "The metrization of rectangular $b$-metric spaces," Topology and its Applications, vol. 261, pp. 22-28, 2019.

[12] M. Abbas and G. Jungck, "Common fixed point results for noncommuting mappings without continuity in cone metric spaces," Journal of Mathematical Analysis and Applications, vol. 341, no. 1, pp. 416-420, 2008.

[13] G. Jungck, "Compatible mappings and common fixed points," International Journal of Mathematics and Mathematical Sciences, vol. 9, no. 4, pp. 771-779, 1986. 


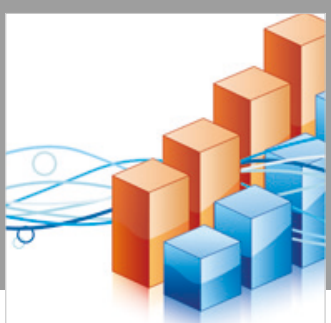

Advances in

Operations Research

\section{-n-m}
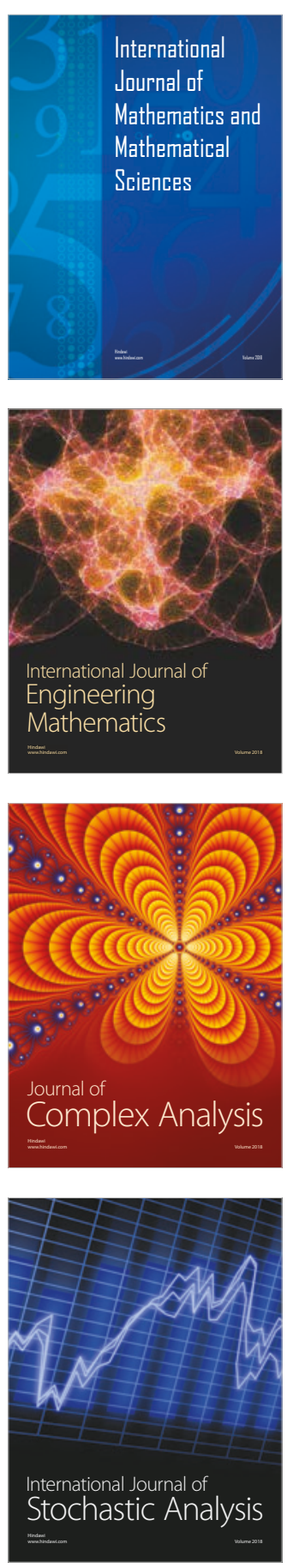
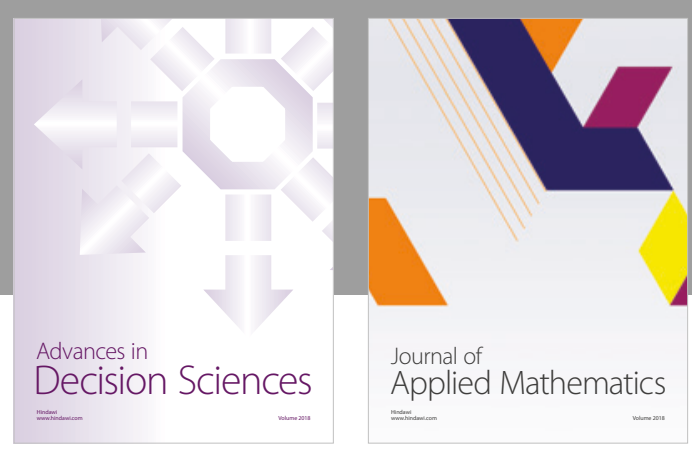

Journal of

Applied Mathematics
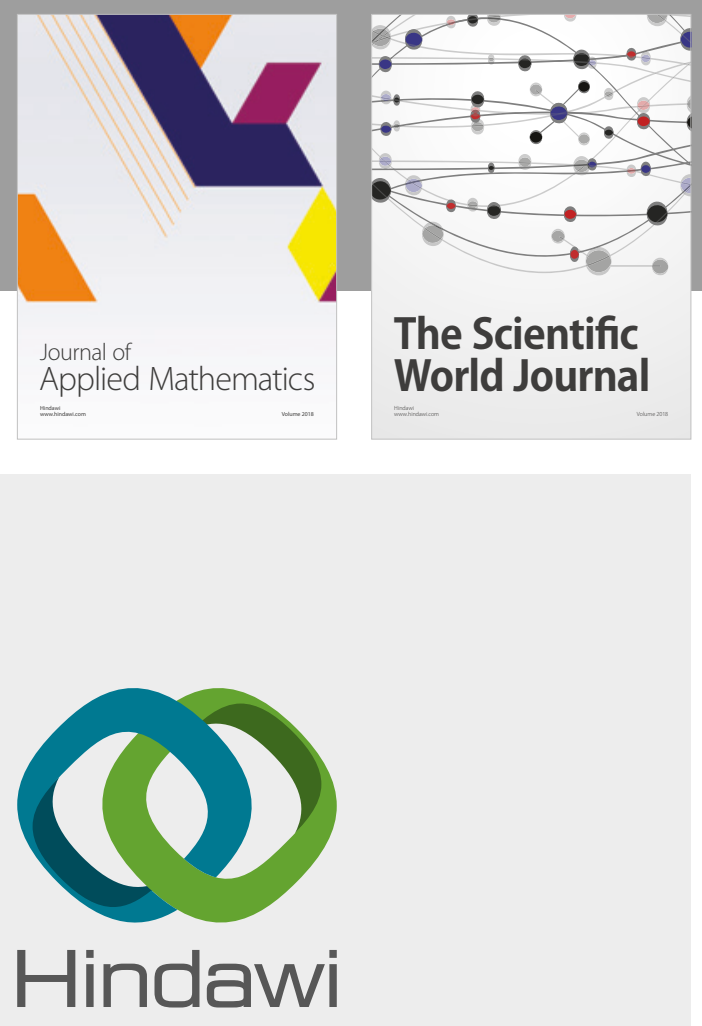

Submit your manuscripts at

www.hindawi.com

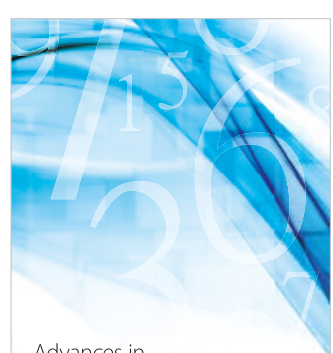

Advances in
Numerical Analysis
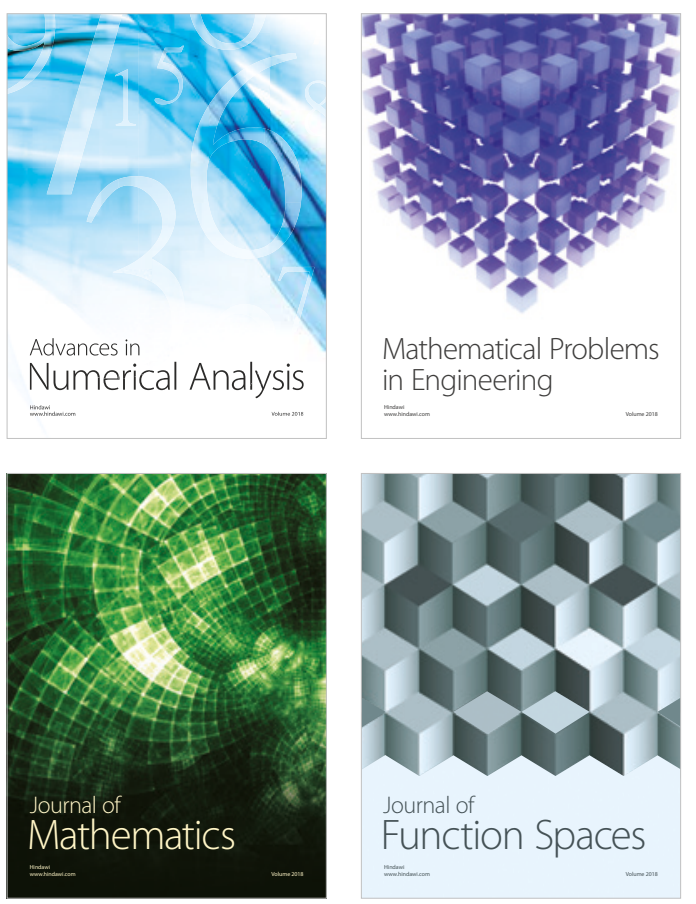

Mathematical Problems in Engineering

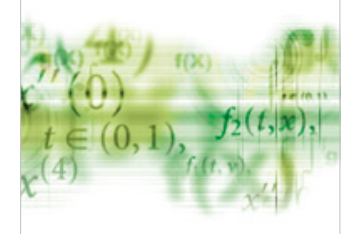

International Journal of

Differential Equations

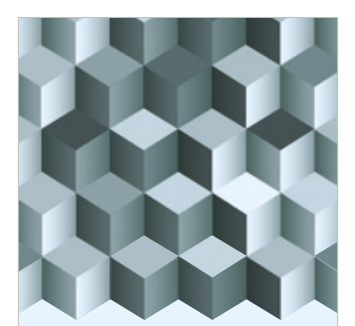

Journal of

Function Spaces

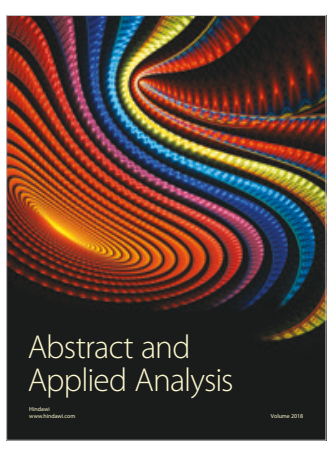

The Scientific

World Journal

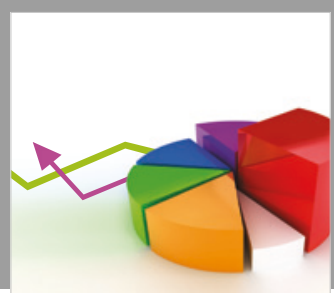

Journal of

Probability and Statistics
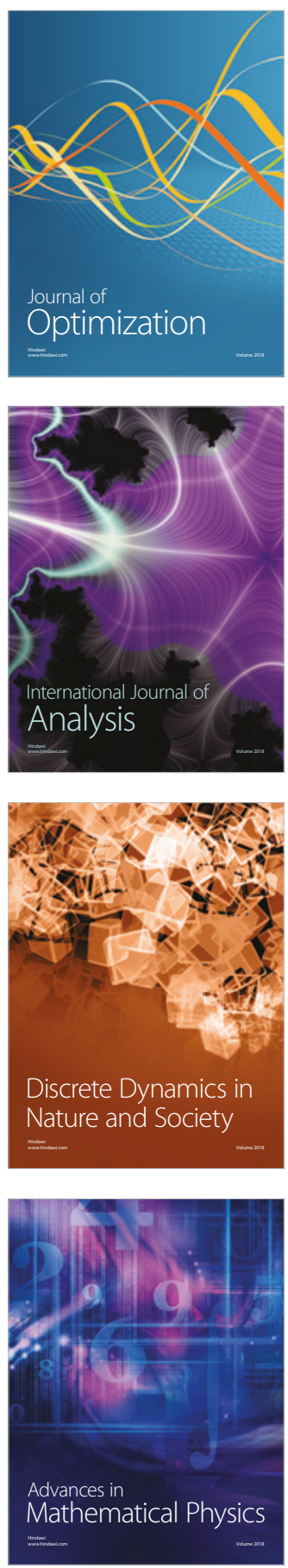